\title{
Primary Care of Adults with Developmental Disabilities in Ontario
}

\author{
Yona Lunsky, Robert S. Balogh, Virginie Cobigo, Barry Isaacs, Elizabeth Lin and Hélène M.J. Ouellette-Kuntz
}

\begin{abstract}
The health status and healthcare of adults with developmental disabilities have not been well-studied in Ontario, due to the absence of population-based data. To address this deficit, the Health Care Access Research and Developmental Disabilities (H-CARDD) program - a provincial partnership of scientists, policymakers and clinicians - has used existing provincial-level administrative data to provide descriptive information on the health of adults with developmental disabilities and the quality of their primary care relative to other adults. H-CARDD's findings have revealed many gaps in the care of adults with developmental disabilities. While primary care providers are critical to achieving needed changes, the broader healthcare context and infrastructure also need to be considered.
\end{abstract}

\section{The Issue}

In recent years, there has been an increased emphasis on the role of primary care in treating vulnerable populations who experience disparities in healthcare. Adults with developmental disabilities, such as Down syndrome or autism, are one such group (Ouellette-Kuntz 2005). They are more likely to have mental health problems (Cooper et al. 2007; Morgan et al. 2008) and physical ailments (van Schrojenstein Lantman-De Valk et al. 2000; Jansen et al. 2004) and to need resourceintensive healthcare than other individuals (Polder et al. 2002). However, because of their cognitive and adaptive impairments, they face unique challenges in navigating their way through an already fragmented healthcare system. This is why their primary care is so important.

Recognizing this need, the first Canadian primary care guidelines to focus on adults with developmental disabilities were published by Sullivan et al. (2006, 2011 [update]). Due to the absence of Canadian data, however, these important guidelines were informed primarily by research from other jurisdictions. From a policy perspective, it is difficult to forecast health needs and target health interventions for adults with developmental disabilities without knowing the size of this population and its key health concerns. Such information is not well-captured in community surveys targeting the general population, and at this time, there are no clinical registries specific to developmental disabilities. One way to address this gap is through existing provincial-level administrative data, which requires the linking of information held by the various government sectors (e.g., health, social services and education) that serve the developmental disabilities population.

\section{The Study}

The Health Care Access Research and Developmental Disabilities (H-CARDD) Primary Care Project is a provincial partnership of scientists, policymakers and clinicians with three goals: to provide prevalence estimates, demographic information and a description of the disease profiles of Ontario adults with developmental disabilities; to examine their healthcare use relative to other adult Ontarians; and to assess how consistent their care was with primary care guidelines.

The project was completed in two stages. The first stage involved creating and describing a province-wide cohort of adults with developmental disabilities aged 18-64 years. This was accomplished by linking social service-based diagnostic information from the Ontario Disability Support Program with administrative health data held at the Institute for Clinical Evaluative Sciences. The second stage consisted of studying the healthcare of this cohort relative to a random sample of $20 \%$ of the Ontario population in the same age range. Aspects of healthcare described included the use of primary care, emergency care and hospitalizations, visits to medical specialists and medication use. To evaluate care, a set of healthcare indicators based on care guidelines were used. These included the periodic health examination; breast, cervical and colorectal cancer screening; eye examinations for individuals with diabetes; care for low trauma fractures; follow-up care for mental health-related emergency visits; avoidable hospitalizations; high-risk medication prescriptions; and the monitoring of multiple medications by primary care physicians. 


\section{Key Findings}

Project findings are summarized in the Atlas on the Primary Care of Adults with Developmental Disabilities in Ontario (Lunsky et al. 2013).

\section{Prevalence, Demographic and Disease Profiles}

In the linked data, 66,484 adults aged 18-64 were coded as having developmental disabilities. This corresponds to a prevalence of $0.78 \%$, an estimate somewhat higher than would be expected based on earlier reports. Compared to adults without developmental disabilities, adults with developmental disabilities were more likely to be younger, male and live in low-income neighbourhoods or rural areas. They were more likely than other adults to be diagnosed with psychiatric disorders, asthma, diabetes, chronic obstructive pulmonary disease and congestive heart failure. A greater proportion of adults with developmental disabilities had scores indicative of high morbidity compared to other adults (24.0\% vs. $15.5 \%)$.

\section{Healthcare Utilization Patterns}

We studied healthcare utilization between April 1, 2009 and March 31, 2010. Despite comparable use of family physicians and similarities in continuity of care, adults with developmental disabilities were more likely than other adults to visit emergency departments (33.9\% vs. $20.2 \%)$ and be hospitalized (7.5\% vs. $4.4 \%)$. They were also more likely to visit at least one specialist $(42.0 \%$ vs. $35.7 \%)$. In particular, they were more likely than other adults to visit a psychiatrist $(14.9 \%$ vs. $3.1 \%)$ or neurologist $(6.0 \%$ vs. $2.1 \%)$.

In the period overlapping October 1, 2009, 60.5\% of adults with developmental disabilities were dispensed at least one medication, with $26 \%$ dispensed two to four medications concurrently and $21.5 \%$ dispensed five or more medications concurrently. The most commonly filled prescriptions were for psychotropics, with $21.1 \%$ of the 52,404 adults being dispensed antipsychotic medications. One concerning high-risk pattern was the dispensing of two antipsychotics concurrently; this was found to occur in $19.1 \%$ of those who filled an antipsychotic medication prescription.

\section{Primary Care Indicators}

In terms of secondary prevention, which is a key responsibility of the primary care physician, only $22.0 \%$ of adults with developmental disabilities received a periodic health examination within two years, and their cancer screening rates were notably lower. Specifically, $32.0 \%$ of adults with developmental disabilities aged 50-64 were up-to-date with colorectal tests compared to $47.2 \%$ of other same-aged adults; $52.2 \%$ of women with developmental disabilities aged 50-64 had received a mammogram in a two-year period compared to $70.7 \%$ of other same-aged women; and $33.7 \%$ of women with developmental disabilities aged 18-64 had cervical cancer screening over a three-year period compared to $66.7 \%$ of other same-aged women.

Although some measures of chronic disease management were similar when comparing adults with developmental disabilities to those without, many results did not meet guideline recommendations. Patients with developmental disabilities and diabetes were more likely to receive eye examinations than patients with diabetes alone (63.2\% vs. 56.9\%). However, they were less likely to receive a bone mineral density test within a year $(16.2 \%$ vs. $21.5 \%)$ even though their rate of low-trauma fractures was nearly three times as high. When individuals with developmental disabilities visited the emergency department for a mental illness, $41.9 \%$ of them did not receive follow-up within 30 days with either a psychiatrist or family physician; this was despite specific guidelines on the importance of crisis follow-up for this population (Sullivan et al. 2006). Medication monitoring also occurred less frequently than recommended (Sullivan et al. 2006); 32.3\% of adults with developmental disabilities who were dispensed five or more medications concurrently did not have regular follow-up visits with the same family physician.

\section{What Do These Findings Mean?}

Compared to other adults, adults with developmental disabilities have more health issues, and these require greater research and clinical attention. While primary care providers are critical to achieving needed changes, the broader healthcare context and infrastructure also need to be considered. Thus, to better meet the healthcare needs of adults with developmental disabilities in Ontario, change needs to focus on three areas:

1. Improving quality of primary care based on best evidence and care standards: Comprehensive primary care for adults with developmental disabilities requires a balanced emphasis on mental and physical health issues and on the prevention and management of chronic disease. Greater awareness of existing evidence-based practice guidelines and the development of clinical competencies are key. The complex care needs of those with developmental disabilities requires an interprofessional approach with an emphasis on embedding guidelines and clinical tools into daily practice. Flagging the presence of developmental disability within the healthcare record with links to appropriate clinical guidelines and tools would be one way to do this.

2. Modifying broader healthcare system structures and processes: Healthcare interventions targeted towards populations with complex needs should include adults with developmental disabilities. This includes focusing on the development and maintenance of care plans, 
fostering collaboration and coordination across the health system and including other relevant sectors. In the current system, many primary care providers do not have sufficient time or support to give the care required to their patients with developmental disabilities (Wilkinson et al. 2012). Excellence in collaborative care is more likely to occur when financial structures supporting such care are put into place.

3. Strengthening partnerships with patients, their families and their paid caregivers: To improve accessibility and quality of care, it is essential that patients be at the centre of that care and that those involved in supporting them, whether paid or unpaid, be recognized for the vital role they play. Their perspective must be considered in the development of effective policies and interventions to improve their health.

\section{Next Steps}

H-CARDD has expanded its primary care focus to study the broader healthcare system and to focus on vulnerable subpopulations of individuals with developmental disabilities, such as women, those with psychiatric disorders, youth transitioning into adulthood and aging adults. We are also evaluating efforts to improve the healthcare of those with developmental disabilities (see <www.hcardd.ca>). HQ

\section{References}

Cooper, S.A., E. Smiley, J. Morrison, A. Williamson and L. Allan. 2007. "Mental Ill-Health in Adults with Intellectual Disabilities: Prevalence and Associated Factors." British Journal of Psychiatry 190(1): 27-35.

Health Care Access Research and Developmental Disabilities (H-CARDD). 2013. Retrieved June 4, 2014. <http://www.hcardd.ca>.

Jansen, D.E., B. Krol, J.W. Groothoff and D. Post. 2004. "People with Intellectual Disability and Their Health Problems: A Review of Comparative Studies." Journal of Intellectual Disability Research 48(2): 93-102.

Lunsky, Y., J.E. Klein-Geltink and E.A. Yates, eds. 2013. Atlas on the Primary Care of Adults with Developmental Disabilities in Ontario. Toronto, ON: Institute for Clinical Evaluative Sciences.

Morgan, V.A., H. Leonard, J. Bourke and A. Jablensky. 2008. "Intellectual Disability Co-Occurring with Schizophrenia and Other Psychiatric Illness: Population-Based Study." British Journal of Psychiatry 193(5): 364-72.

Ouellette-Kuntz, H. 2005. "Understanding Health Disparities and Inequities Faced by Individuals with Intellectual Disabilities." Journal of Applied Research in Intellectual Disabilities 18(2): 113-21.
Polder, J.J., W.J. Meerding, L. Bonneux and P.J. Maas. 2002. "Healthcare Costs of Intellectual Disability in the Netherlands: A Cost-of-Illness Perspective." Journal of Intellectual Disability Research 46(2): 168-78.

Sullivan, W.F., J. Heng, D. Cameron, Y. Lunsky, T. Cheetham, B. Hennen et al. 2006. "Consensus Guidelines for Primary Health Care of Adults with Developmental Disabilities." Canadian Family Physician 52(11): 1410-18.

Sullivan, W.F., J.M. Berg, E. Bradley, T. Cheetham, R. Denton, J. Heng et al. 2011. "Primary Care of Adults with Developmental Disabilities: Canadian Consensus Guidelines." Canadian Family Physician 57(5): 541-53.

van Schrojenstein Lantman-De Valk, H.M.J., J.F.M. Metsemakers, M.J. Haveman and H.F.J.M. Crebolder. 2000. "Health Problems in People with Intellectual Disability in General Practice: A Comparative Study." Family Practice 17(5): 405-07.

Wilkinson, J., D. Dreyfus, M. Cerreto and B. Bokhour. 2012. "'Sometimes I Feel Overwhelmed': Educational Needs of Family Physicians Caring for People with Intellectual Disability." Intellectual and Developmental Disabilities 50(3): 243-50.

\section{About the Authors}

Yona Lunsky, PhD, CPsych, is a clinician scientist at the Centre for Addiction and Mental Health and an adjunct scientist at ICES. She is an associate professor in the Faculty of Medicine at the University of Toronto.

Robert S. Balogh, PhD, BHSc(PT), is an assistant professor in the Faculty of Health Sciences at the University of Ontario Institute of Technology and an adjunct scientist at ICES.

Virginie Cobigo, PhD, CPsych, is an assistant professor in the School of Psychology and a senior researcher at the Centre for Research on Educational and Community Services at the University of Ottawa. She is an adjunct scientist at ICES.

Barry Isaacs, PhD, is Director of Research, Evaluation and Education at Surrey Place Centre in Toronto.

Elizabeth Lin, PhD, is an independent scientist in the Provincial System Support Program at the Centre for Addiction and Mental Health and an adjunct scientist at ICES. She is an assistant professor in the Faculty of Medicine at the University of Toronto.

Hélène M.J. Ouellette-Kuntz, PhD, RN, is a professor in the Department of Public Health Sciences at Queen's University, an epidemiologist at Ongwanada and an adjunct scientist at ICES. 